Artículo Original/Original Article

\section{Construcción del vínculo terapéutico entre odontólogo y paciente}

\section{Construction of the therapeutic relationship between dentist and patient}

\author{
Marega $\mathrm{GH}^{1}$., Sanchez Dagúm $\mathrm{M}^{1}$. \\ ${ }^{I}$ Universidad Nacional de Córdoba, Facultad de \\ Odontología, Departamento de Preventiva y Social. Cátedra \\ de Odontología Preventiva y Comunitaria I.
}

\section{Resumen}

El vínculo terapéutico es un compromiso o alianza interpersonal entre paciente y profesional, el objetivo del presente trabajo fue valorar aspectos del vínculo que se construye entre los alumnos que cursan las practicas clínicas del último año de la carrera de Odontología y sus pacientes, revalorizando la importancia de la relación interpersonal que se establece con motivo de esta atención debido al valor que ésta tiene en el tratamiento delos mismos. Método. se llevó a cabo un estudio cuanti-cualitativo, observacional, descriptivo y transversal, realizado en dos fases: cuantitativa, a través del diseño y aplicación de instrumentos de recolección de datos y su posterior análisis estadístico mediante frecuencia absoluta y relativa; de acuerdo a los estudios de recuento de casos, media, mediana y rango, según los métodos no paramétricos de contraste. Y cualitativa, mediante el abordaje etnográfico realizado en dos cátedras de la Facultad de Odontología, la observación de los datos registrados en una matriz, la interacción entre los mismos y la reflexión que se hace sobre ellos. Ambas, sobre muestras conformadas por alumnos que cursan el Ciclo Profesional y pacientes por ellos asistidos en las prácticas clínicas obligatorias de la carrera de grado en la institución. Los resultados obtenidos muestran que las dimensiones propuestas para alumnos (características sociodemográficas, relación alumno-paciente) y para pacientes (características socio-demográficas de la población, motivación de la demanda, calidad de la atención recibida) se relacionaron con las categorías cualitativas (contexto, actividades, participación y sus subcategorías). Conclusión: el vínculo con el paciente es fundamental en relación al éxito del tratamiento es necesario que los alumnos profundicen aspectos relacionados con estos temas desde lo curricular.

PALABRAS CLAVE: Odontólogo, Paciente, Relación, Estudiante de Odontología.

\begin{abstract}
The therapeutic link is a commitment or interpersonal alliance between patient and professional, the objective of this work was to assess aspects of the bond that is built between the students who attend the clinical practices of the last year of the career of Dentistry and their patients, revaluing the importance of the interpersonal relationship that is established because of this attention due to the value that it has in the treatment of them. Method. a qualitative, observational, descriptive and cross-sectional study was carried out, carried out in two phases: quantitative, through the design and application of data collection instruments and their subsequent statistical analysis by absolute and relative frequency; according to the studies of case count, mean, median and range, according to non-parametric contrast methods. And qualitative, through the ethnographic approach carried out in two chairs of the Faculty of Dentistry, the observation of the data recorded in a matrix, the interaction between them and the reflection that is made about them. Both, samples formed by students who attend the Professional Cycle and patients assisted by them in the compulsory clinical practices of the degree program at the institution. The results obtained show that the proposed dimensions for students (socio-demographic characteristics, student-patient relationship) and for patients (sociodemographic characteristics of the population, demand motivation, quality of care received) were related to the qualitative categories (context, activities, participation and its subcategories). Conclusion: the link with the patient is fundamental in relation to the success of the treatment it is necessary that the students deepen aspects related to these topics from the curricular.
\end{abstract}

KEY WORDS: Dentist, Patient, Relationship, Student of Dentistry

\section{Introducción}

Un vínculo (etimológicamente del latín "vínculum", deriva de "vincre": atar, lazo, ligadura) ${ }^{1}$ significa algo que une una cosa a otra. 
Para la Psicología es la vivencia corporal, psíquica e intelectual de la relación hacia otras personas; el vínculo terapéutico es un compromiso o alianza interpersonal entre paciente y profesional ${ }^{2}$.

En la relación paciente-profesional, se establece un campo de interrelación emocional, donde se desarrolla la función del profesional: por un lado asistencial, teniendo en cuenta, los conocimientos adquiridos y la técnica de su especialidad clínica, y por el otro, su condición humana, que siente, se emociona, sufre y que es impactado por la presencia de otro ser humano que lo solicita ${ }^{3}$.Esta relación es asimétrica, según Arrow ya que el profesional tiene un cuerpo de conocimientos de los que el paciente suele estar excluido ${ }^{4}$.

Los aportes realizados respecto a "relaciones interpersonales de pacientes y profesionales de las Ciencias de la Salud", arrojan en sus conclusiones, que las mismas constituyen una cuestión central para el éxito del tratamiento y que si bien se reconocen como un aspecto beneficioso para los vínculos entre ambos, en la práctica profesional diaria son escasas las estrategias o mecanismos que se consideran para establecer estas relaciones y/o mejorar las mismas. La construcción de una relación afectiva profunda y confiada con el profesional incrementa las potencialidades asistenciales, elevando los efectos de todos los recursos terapéuticos y rehabilitadores a utilizar con el objetivo de curar, evitar discapacidad y mitigar sufrimientos ${ }^{5}$. Este tema ha despertado un renovado interés en la producción científica, la formación y la práctica clínica buscando ofrecer una mejor calidad en esta relación.

Durante las últimas décadas, las Instituciones Formadoras en Ciencias de la Salud han considerado que, para que el Plan de Estudios tenga un perfil adecuado, el tema de la relación "profesional de la salud - paciente" debe estar presente durante todo el proceso de formación de los estudiantes. Mostrando gran interés acerca de la inserción de enfoques interdisciplinarios y la introducción de cambios que buscan la adquisición de competencias respecto a prácticas para la comunicación, la observación, el trabajo en equipo y las actitudes de base en valores humanitarios sólidos ${ }^{6}$. Este proceso se debe ir profundizando progresivamente en contenidos y en habilidades prácticas que llevan a considerar no sólo los aspectos físicos sino también otras características culturales y socioeconómicas; revalorizando la importancia de los pacientes como beneficiarios de los servicios sanitarios prestados ${ }^{7}$.

Según Beltrán Neira (2010), los logros en el futuro ejercicio profesional de nuestros estudiantes dependen de numerosos factores entre los que están incluidas las competencias que constituyen el perfil propuesto por la Institución Formadora.

En este contexto, es apropiada una formación de grado en la Profesión Odontológica que capacite tanto a nivel formativo, como en aspectos éticos, consentimientos informados, protocolos claros de atención para protección de los pacientes, como también en una adecuada comunicación interpersonal. La ética propuesta por las nuevas condiciones de la vida y la técnica debe estar basada en responder ante las demás personas y ante nuestra propia conciencia por nuestros actos; el aporte de la bioética como campo interdisciplinario en el estudio de problemas complejos, la comprensión del hombre, su vulnerabilidad ante la enfermedad, el acrecentamiento de nuestra responsabilidad social y su rol en los diferentes ámbitos de la Odontología, debe tenerse en cuenta para nuestras puestas en práctica, no solo por la conveniencia de obtener resultados más satisfactorios en el futuro ejercicio profesional sino también por convicción ${ }^{8}$.

El propósito de este estudio fue valorar aspectos presentes en la construcción del vínculo y la relación que se establece entre los alumnos y pacientes, su influencia en los procesos y resultados, y en el logro de la salud de los pacientes, en la práctica clínica de la carrera de grado, Facultad de Odontología, UNC.

El presente trabajo forma parte dela tesis Doctoral"Impacto del Vínculo Estudiantepaciente en la Práctica Clínica de Grado".Agosto de 2017. 


\section{Métodos}

El estudio realizado fue cuanti-cualitativo, observacional, descriptivo y transversal, y se llevó a cabo en dos fases.En la primera, se siguió el método cuantitativo, mediante la aplicación de dos encuestas, individuales y semi-estructuradas, a alumnos y pacientes, para conocer "qué" y "cuánto", aplicando el método hipotético deductivo $\mathrm{y}$, posteriormente, análisis estadístico de los datos. Es el procedimiento o camino seguido por un investigador para hacer de su actividad una práctica científica; este método obliga al científico a combinar la reflexión racional (la formulación de hipótesis y la deducción) con la observación de la realidad o momento empírico (la observación y la verificación). El pensamiento va de lo general a lo particular.

En la segunda fase, se siguió la metodología cualitativa para conocer el "porqué" de la situación, utilizando el razonamiento inductivoanalítico y tratando de captar los hechos en su contexto.

La población en estudio estuvo compuesta por los alumnos (Población A) que cursan el Ciclo Profesional, aproximadamente de 298 miembros $(\mathrm{N})^{9}$ y los pacientes que ellos atienden (población B), en las prácticas clínicas obligatorias de la carrera de grado en la Facultad de Odontología de la UNC. La muestra obtenida de la Población A, se obtuvo mediante un muestreo aleatorio simple, considerando una proporción media de $25 \%$ de alumnos, un nivel de significancia de $\alpha=0,05$ y una eficiencia del $95 \%, n=71$.

Los instrumentos para la recolección de datos, fueron dos encuestas individuales semiestructuradas, dirigidas a las muestras alumnos y pacientes, de contenido multidimensional, dicotómicas, tricotómicas y de respuesta múltiple, las preguntas incluyeron dimensiones y variables con sus respectivas categorías de estudio diseñadas ad hoc. Ambos instrumentos fueron validados por expertos y por pares de acuerdo a su de criterio y contenidos, y aprobado por Comité de Ética de Investigación en Salud CIEIS- (2013).

En la fase cualitativa, se recolectaron datos sobre la relación establecida por los pacientes y alumnos seleccionados en el cursado del Ciclo Profesional, pertenecientes a las cátedras de Práctica Profesional Supervisada y Prostodoncia IV “B”, según muestreo teórico.

El método empleado fue el de observación en terreno. Se llevó a cabo a través del abordaje etnográfico como estrategia cualitativa de investigación social, el cual es una herramienta que permite tomar contacto directo con los actores seleccionados; el objetivo es recolectar datos directamente de los contextos y situaciones observadas, lo que posibilita elaborar descripciones de las acciones, discursos e intercambios que tienen lugar entre los actores.Se trabajó con el instrumento "observación directa a alumnos, pacientes y docentes".

Para la observación se tuvo en cuenta la captación de los fenómenos y de los sucesos que se relacionaban con los aspectos cognitivos, actitudinales y prácticos.

\section{Resultados}

Los porcentajes expresados en la Tabla 1 reflejan los aspectos negativos según sexo y pruebas de $\mathrm{Chi}^{2}$ de Pearson, percibidos por los alumnos en relación al trato del paciente.

En el primer aspecto evaluado, "ansiedad", se verificó una diferencia significativa entre mujeres y varones $(\mathrm{p}<0,05)$; el riesgo relativo $(\mathrm{RR}) \mathrm{de}$ ansiedad en mujeres es 3,4 veces mayor que en varones (IC 95\% 1,155- 10,051). La mayoría indicó que necesita adquirir más seguridad en el trato hacia el paciente $(51,5 \%)$, particularmente los varones $(61,9 \%)$. El $41 \%$ de los alumnos tiene un enfoque casi exclusivamente técnicocientífico, información que resulta de gran relevancia para el presente estudio. 
En la Tabla 2, se observan porcentajes descriptivos de percepción de los alumnos en relación a la participación del docente. Para complementar la información anterior respecto de imitar el desempeño del docente (conocimientos, competencias, destrezas y técnicas), se presenta esta tabla en la que se expresan, en orden decreciente de frecuencia, las situaciones mencionadas como ejemplo por los alumnos.

En relación al éxito del tratamiento y el vínculo establecido con el paciente, la Tabla 3 muestra que: todos los alumnos consideraron importante $o$ muy importante el vínculo con el paciente en relación al éxito del tratamiento. Específicamente, el 77,5\% lo consideró "muy importante". Las diferencias porcentuales entre sexos no resultaron estadísticamente significativas $(p>0,05)$.

Considerando los motivos por los que el alumno considera importante el vínculo con el paciente en relación al éxito del tratamiento, con referencia a la pregunta de la encuesta: "¿Crees que tus prácticas en general han sido beneficiosas para los pacientes? ¿Por qué?”,se refleja en el recuento numérico expresado en la Tabla 4 el número de alumnos que considera importante el vínculo establecido con su paciente. Cabe aclarar que se podía elegir más de una opción.

Para llevar a cabo sus prácticas clínicas, la gran mayoría de los alumnos, el 97,2\%, capta a sus pacientes mediante búsqueda propia.

Como se expresa en la Tabla 5 y 6 , respecto a las opciones de captación, es lógico que el porcentaje predominante refleje el modo más favorable desde el punto de vista del vínculo.

\section{Discusión}

En nuestra investigación, se planteó como variable el "cumplimiento de la ética por parte de los alumnos",la mayor parte de ellos (76,8\%) consideraron importante respetar la privacidad del paciente, como también sintieron que estaban realizando una práctica beneficiosa, lo que da cuenta de su adhesión a los principios éticos de secreto profesional, beneficencia, no maleficencia y autonomía. Fodor A, y de la Parra
G, a través de estudios realizados en la Universidad de Chile, sostienen la importancia de la preparación odontológica a nivel formativo en: aspectos éticos, consentimientos informados y protocolos de atención para protección de pacientes y profesionales ${ }^{10}$.

López Portilla señala que los pacientes atendidos en la clínica odontológica de la Universidad de Antioquia, identificaron como una de las principales fortalezas del servicio, la relación establecida entre los docentes y los estudiantes; el trato que reciben de los estudiantes, reflejado en el interés por escuchar sus necesidades y el deseo de atenderlos ${ }^{11}$. Para nuestro estudio, los aspectos del alumno más ponderados por los pacientes fueron: el respeto y la confianza, para las pacientes mujeres; la responsabilidad y la escucha, para los pacientes varones.

La preparación del alumno fue el de menor frecuencia; sólo $23(32,9 \%)$ pacientes eligieron esta opción. Por otra parte, al valorar la importancia del vínculo con el paciente en relación al éxito del tratamiento, todos los alumnos indagados la consideraron importante $o$ muy importante.

Varela et al. en un estudio multicéntrico realizado en la Facultad de Odontología de la Universidad Católica de Córdoba, manifiestan que: "es importante conocer los elementos que promueven la orientación empática entre estudiantes y pacientes, en la situación de atención clínica durante el transcurso de la carrera y relacionarla con estrategias formativas que permitan mejorar este nivel".

Señalando también "que las mujeres son más empáticas que los hombres al valorar la diferencia en los niveles de orientación empática, según sexo"2.

En nuestros resultados, al evaluar los aspectos percibidos por los alumnos en su relación con el paciente, clasificados por géneros, se verificó una diferencia significativa entre mujeres y varones en el nivel de ansiedad, que resultó 3,4 veces mayor en las primeras.

La mayoría de los alumnos encuestados indicó que necesita adquirir más seguridad en la relación 
con el paciente, particularmente los varones $(61,9 \%)$.

Mandozzi. en su tesis doctoral, expresa que " $\mathrm{el}$ trato otorgado por los prestadores constituye un elemento fundamental en la satisfacción de las expectativas, y si los pacientes no perciben un trato amable las expectativas de satisfacción se ven afectadas negativamente" 13 .

En nuestra investigación, el $41 \%$ de los alumnos, de ambos sexos, percibe tener un enfoque casi exclusivamente técnico-científico al realizar sus prácticas, información que resulta de gran relevancia para el presente estudio.

En el presente estudio, al indagar a alumnos y pacientes por las vías y fluidez en la comunicación, dejaron de manifiesto las dificultades prácticas que esto supone en el ámbito público universitario. Lo cual no es ideal si se pretende formar profesionales comprometidos, con un perfil más humanitario.

Según Restrepo, es necesario que el profesional se apropie de habilidades que vayan conformando una cultura de la comunicación, con la finalidad de promover cambios y lograr aprendizajes conscientes que conduzcan a la población a modificar conductas y estilos de vida $^{14}$.

Este proyecto pretende revalorizar la importancia de la relación interpersonal que se establece con motivo de la atención odontológica, debido al valor que ésta tiene en el tratamiento del paciente, en relación a la conservación y/o recuperación de su salud, mediante la promoción de prácticas de autocuidado y la adopción de conductas saludables.

\section{Agradecimientos}

Al Profesor Dr. Nelson Rugani y Profesor Dr.Agustín Villa por permitirnos realizar las actividades de campo en las cátedras de Prostodoncia IV "B" y Práctica Profesional Supervisada. Al Ing. Luis Croharé por su participación en el trabajo estadístico de este estudio.

\section{Referencias}

1. De Castro L. Odontología: Ciencia y Arte. Acta Odontol Venez. 2006; 44(3)80-81.
2. Beltrán-Neira RJ. Evaluación de la enseñanza aprendizaje. Certificación de competencias. Rev Estomatol Herediana. 2010 20(2):112-113.

3. Mandozzi M. Valoración del Grado de Satisfacción de los Pacientes Asistidos en los Servicios Odontológicos de Atención Externa de la Facultad de Odontología, Universidad Nacional de Córdoba [tesis doctoral]. 2010. Escuela de Posgrado, Facultad de Odontología, UNC.

4. Plan de Estudios Carrera de Odontología, UNC. Año 2010. Ord. HCD 4/2010.

5. Garzón Maceda F. Universidad Nacional de Córdoba: Historia de la Facultad de Ciencias Médicas. 1927.At: www.fcm.unc.edu.ar/biblio. Último acceso: Septiembre 18, 2015.

6. Sánchez Dagúm M., Sánchez Dagúm E, Visvisian C. La problemática desde la colonia hasta la actualidad: equidad y Salud desde una perspectiva interdisciplinaria: La formación académica y el ejercicio profesional de la Odontología, dos siglos de historia [CD-ROM]. 2010. Ponencia Bicentenario. UNC.

7. Perfil del Odontólogo.Facultad de Odontología, UNC. At:

http://www.odo.unc.edu.ar/files/Plan_de_Estudios_Odo ntologia_2011.pdf. Último acceso: Noviembre 24, 2016.Ibid 3

8. Fuente de Información Centro de Cómputos de la Facultad de Odontología, UNC. 2011.

9. Fodor A, De la Parra G. La Relación Paciente/Odontólogo: Algunas Consideraciones. Rev. $\begin{array}{lllll}\text { Chil. } 2007 . & 99 & \text { (3) 21-26. At: }\end{array}$ http://www.revistadentaldechile.cl/.Último acceso: Marzo 4 de 2015.

10. López-Portilla J, et al. Determinantes de la satisfacción de la atención odontológica en un grupo de pacientes atendidos en la Clínica del Adulto de la Facultad de Odontología de la Universidad de Antioquia. Gerencia y Políticas de Salud, 12(24). 2013. At: http://revistas.javeriana.edu.co/index.php.Último acceso: Julio 8, 2016.

11. Varela $\mathrm{T}$, et al. Niveles de orientación empática en estudiantes de Odontología de la Universidad Católica de Córdoba, Argentina (UCC). 2012 (mayo de 2015), 19(70):15-22. At: www.bases.bireme.br.Último acceso: Septiembre 15, 2016. Ibid 3

12. Restrepo E. Técnicas Etnográficas. Etnografías Contemporáneas 1 (1): 162-179. 2015. At: http://www.unsam.edu.ar/revistasacademicas/index.php 
/etnocontemp/article/viewFile/21/13. Último acceso: Octubre 28, 2015.

\section{Correspondencia a/ Corresponding to:}

Dra. Gabriela H. Marega.

Domicilio postal: Félix Frías $274 B^{\circ}$ General Paz.

Correo electrónico/E-mail: gabimarega@ yahoo.com.ar. 
Tabla 1. Percepción de los alumnos en relación al trato con el paciente

\begin{tabular}{|l|c|c|c|c|}
\hline Aspecto negativo evaluado & Total & Mujeres & Varones & $\begin{array}{c}\mathbf{C h i}^{\mathbf{2}} \\
(p \text {-valor })\end{array}$ \\
\hline Se siente ansioso (si) & $\mathbf{4 0 . 0 \%}$ & $51.1 \%$ & $15.0 \%$ & 0.006 \\
\hline Necesita mayor seguridad en el trato $(\mathbf{s i})$ & $\mathbf{5 1 . 5 \%}$ & $46.7 \%$ & $61.9 \%$ & 0.249 \\
\hline Se siente naturalmente seguro (no) & $\mathbf{2 8 . 8 \%}$ & $34.8 \%$ & $15.0 \%$ & 0.103 \\
\hline Se enfoca sólo en lo técnico-científico $(\mathbf{s i})$ & $\mathbf{4 1 . 0 \%}$ & $38.6 \%$ & $47.1 \%$ & 0.549 \\
\hline Se siente preparado por sus conocimientos $(\mathbf{n o})$ & $\mathbf{2 5 . 0 \%}$ & $20.8 \%$ & $35.0 \%$ & 0.219 \\
\hline
\end{tabular}

Tabla2: Privacidad de la información suministrada por el paciente

\begin{tabular}{|l|c|c|}
\hline Grado de importancia & $\mathbf{n}$ & $\mathbf{\%}$ \\
\hline Poco importante & 1 & $1.4 \%$ \\
\hline Importante & 24 & $33.8 \%$ \\
\hline Muy importante & 46 & $64.8 \%$ \\
\hline Total & $\mathbf{7 1}$ & $\mathbf{1 0 0 . 0 \%}$ \\
\hline
\end{tabular}

Tabla 3: Relación entre el éxito del tratamiento y el vínculo establecido con el paciente

\begin{tabular}{|l|c|c|c|c|}
\hline Grado de importancia considerado & Total & Mujeres & Varones & Chi $^{\mathbf{2}}($ p-valor $)$ \\
\hline Poco importante & $\mathbf{0 . 0 \%}$ & $0.0 \%$ & $0.0 \%$ & \multirow{2}{*}{0.979} \\
\cline { 1 - 4 } Importante & $\mathbf{2 2 . 5 \%}$ & $22.4 \%$ & $22.7 \%$ & \\
\cline { 1 - 4 } Muy importante & $\mathbf{7 7 . 5 \%}$ & $77.6 \%$ & $77.3 \%$ & \\
\hline
\end{tabular}

Tabla 4: Recuento de motivos

\begin{tabular}{|l|l|}
\hline Motivos & n \\
\hline $\begin{array}{l}\text { Adopta una actitud más participativa, con predisposición en la curación y recuperación } \\
\text { de su salud. }\end{array}$ & 49 \\
\hline $\begin{array}{l}\text { El paciente se siente más comprometido con el tratamiento propuesto, no abandonando } \\
\text { el mismo. }\end{array}$ & 41 \\
\hline Es más fácil que entienda lo que deseamos transmitirle, se agiliza la comunicación. & 32 \\
\hline
\end{tabular}


Tabla 5: Captación de pacientes para prácticas

\begin{tabular}{|l|l|l|}
\hline \multicolumn{2}{|l|}{ Captación de pacientes } & Porcentaje \\
\hline \multirow{3}{*}{ Búsqueda propia } & Amigos, familiares, etc. & $90,1 \%$ \\
\cline { 2 - 3 } & Aviso publicitario & $74,6 \%$ \\
\cline { 2 - 3 } & Centro de Salud & $29,6 \%$ \\
\hline Recepción en F.O. & $19,7 \%$ \\
\hline D. Espontánea en F.O. & $14,1 \%$ \\
\hline Otros & $11,3 \%$ \\
\hline
\end{tabular}

Tabla 6: Relación entre las fases cuantitativa y cualitativa

\begin{tabular}{|c|c|c|}
\hline \multirow{2}{*}{$\begin{array}{l}\text { Categorías y sub- categorías } \\
\text { cualitativas }\end{array}$} & \multicolumn{2}{|c|}{ Dimensiones y variables cuantitativas } \\
\hline & Alumnos & Pacientes \\
\hline $\begin{array}{l}\text { Ambiente-Contexto } \\
\text { *Preparar- anticiparse } \\
\text { *Sala clínica }\end{array}$ & \multirow{3}{*}{$\begin{array}{c}\text { Relación alumno-paciente } \\
\text { *Captación del paciente } \\
\text { *Formación académica: aspectos } \\
\text { objetivos - subjetivos } \\
\text { *Cumplimiento de la ética } \\
\text { *Habilidades comunicativas: } \\
\text { interpersonal u otras. } \\
\text { *Continuidad del vínculo }\end{array}$} & \multirow{3}{*}{$\begin{array}{c}\text { Calidad de la Atención recibida } \\
\text { *Trato recibido durante la atención. } \\
\text { *Valoración de la atención recibida en } \\
\text { relación a la mejora de su Salud. } \\
\text { *Información receptada sobre cuidados } \\
\text { de Salud Bucal. } \\
\text { *Grado de comprensión de las } \\
\text { indicaciones recibidas. }\end{array}$} \\
\hline $\begin{array}{l}\text { Actividades } \\
\text { *Trabajo con el paciente } \\
\text { *Bioseguridad } \\
\text { *Ética }\end{array}$ & & \\
\hline $\begin{array}{l}\text { Participación } \\
\text { *Diálogo } \\
\text { *Relación alumno- } \\
\text { paciente. }\end{array}$ & & \\
\hline
\end{tabular}

